\title{
Mehrsprachigkeitsforschung an der Alpen-Adria-Universität (und darüber hinaus): Eine prospektive Bestandsaufnahme*
}

\author{
Luca MelChior \\ University of Klagenfurt (Austria) \\ $\square$ \\ 0
}

\section{Einleitung}

Mehrsprachigkeit als genuin menschliches Phänomen und Mehrsprachigkeitsforschung als wissenschaftliche Beschäftigung mit den vielfältigen Manifestationen dieses Phänomens kommen in der gegenwärtigen Gesellschaft und in der akademischen Welt eine beständig steigende Aufmerksamkeit zu. Die Renaissance der Dialekte und bisher minoritärer Sprachen und deren (Wieder-)Aufwertung als kulturelles Gut, ${ }^{1}$ das Bekenntnis der Europäischen Union zur Sprachenvielfalt und der Förderung der Mehrsprachigkeit ${ }^{2}$ einerseits, die migratorischen Bewegungen in einer globalisierten Gesellschaft, welche die Idee des Nationalstaates in Frage stellen, die Behauptung des Englischen als Lingua

\footnotetext{
* Der erste Teil dieses Aufsatzes (Kapitel 1-5) basiert auf dem Text meiner Antrittsvorlesung an der AlpenAdria-Universität Klagenfurt/Celovec am 25.06.2019.

1 Diese bringt in vielen Fällen gesetzlich sanktionierte Förderung und Schutz mit sich. Dazu seien die Verabschiedung der Europäischen Charta der Regional- und Minderheitensprachen durch den Europarat im Jahre 1992 sowie deren Unterzeichnung und (teilweise) Ratifizierung in vielen europäischen Staaten in den Folgejahren (dazu vgl. Lebsanft/Wingender 2012), sowie die daraus erfolgenden gesetzlichen Maßnahmen, welche in den einzelnen Staaten unternommen wurden (ein Beispiel dafür ist das italienische Gesetz 482 aus dem Jahre 1999 Norme in materia di tutela delle minoranze linguistiche storiche) genannt.

2 So wird im Artikel 2 des Vertrags von Lissabon (2007) festgehalten, dass die EU „den Reichtum ihrer kulturellen und sprachlichen Vielfalt [wahrt] und sorgt für den Schutz und die Entwicklung des kulturellen Erbes Europas“; schon im Jahre 2002 wurde im Beschluss von Barcelona des EU-Ministerrats das Ziel „Muttersprache plus zwei Fremdsprachen“ für jede*e EU-Bürger*in beschlossen (Kommission der europäischen Gemeinschaften 2007), deren Umsetzung jedoch noch weit entfernt ist.
}

(c) by the author; luca.melchior@aau.at

Colloquium: New Philologies, Volume 5, Issue 2 (2020)

doi: 10.23963/cnp.2020.5.2.1

Stable URL: https://colloquium.aau.at/index.php/Colloquium/article/view/135

This work is licensed under a Creative Commons Attribution 4.0 International License (CC BY 4.0). 
franca und die teils erkennbare Reduzierung der Mehrsprachigkeit auf den Erwerb von Englischkompetenzen (vgl. kritisch dazu Trabant 2018; Lüdi 2011, 18), aber auch die steigende Bedeutung von anderen auf dem Welt(sprachen)markt, wertvollen' Sprachen und die daraus folgende commodification of language andererseits sind nur einige der Aspekte, die ein gesteigertes Interesse gegenüber Mehrsprachigkeit bedingen. Gleichzeitig stehen die genannten Aspekte aber stellvertretend für unterschiedliche Funktionen, welche Sprachen annehmen können, nämlich symbolische, identitätsstiftende einerseits, instrumentelle andererseits (vgl. u.a. Coulmas 2005, 11) und illustrieren dadurch schon die Breite und Vielfalt des Gegenstands und möglicher sich darauf beziehender Forschungsinteressen. Im vorliegenden Artikel wird zunächst eine kurze historische Übersicht über die Entwicklung des wissenschaftlichen Interesses an Mehrsprachigkeit gegeben. Daran anschließend werden einige der außersprachlichen Faktoren und Umstände illustriert, die als Erklärungsmoment für diese Entwicklung gesehen werden können sowie ausgewählte zentrale Themen der Mehrsprachigkeitsforschung umrissen. Nach einer vor diesem Hintergrund profilierten Zusammenschau von unterschiedlichen, aber durchaus komplementären Theorien über Sprache und Sprachlichkeit werden schließlich Impulse für eine Verankerung der Mehrsprachigkeitsforschung - u.a. unter Rückgriff auf die Erfahrungen an der Alpen-Adria-Universität Klagenfurt/Celovec - vorgeschlagen. Daran anschließend werden die Beiträge dieser Ausgabe, welche einen Ausschnitt der an unserer Universität stattfindenden Forschung zur Mehrsprachigkeit zeigen, vorgestellt.

\section{Mehrsprachigkeit: ein interdisziplinäres Forschungsfeld gewinnt an Bedeutung}

Obwohl schon im 19. Jahrhundert bahnbrechende Arbeiten zum Sprachkontakt und zur Sprachmischung entstanden sind - man denke z.B. an die Arbeiten von Miklosich (es sei hier nur Miklosich 1889 erwähnt) oder an die Kreolistik und an das Werk Hugo Schuchardts $^{3}$ gewinnt die Mehrsprachigkeitsforschung erst mit Uriel Weinreichs Languages in Contact ([1953] 1968) an Sichtbarkeit, wird dann nach und nach in der zweiten Hälfte des 20. Jahrhunderts von einem randständigen Forschungsbereich zu einem der zentraleren. So schreiben die Herausgeber*innen in der Einleitung zu The Routledge Handbook of Multilingualism (Martin-Jones, Blackledge und Creese 2012a):

\footnotetext{
3 Es ist hier müßig, ein Werk von Schuchardt exemplarisch anzuführen, steht doch fast sein gesamtes Oeuvre im Zeichen der Sprachkontaktforschung.
} 
The last two decades have seen a rapidly growing interest, internationally, in multilingualism and multilin-gual literacy and in the ways in which multilingualism is represented in the media and in public discourse. This is largely due to the significant linguistic, cultural and demographic changes that have been ushered in by globalization, transnational population flows, the spread of new technology and the changing political and economic landscape of different regions of the world.

The last two decades have also seen the emergence of new strands of research on multilingualism, which have incorporated critical and post-structuralist perspectives from social theory and embraced new episte-mologies and research methods. There has also been a shift of focus to empirical work, which is interpretive, ethnographic and multimodal in nature. (Martin-Jones, Blackledge und Creese 2012b, 1)

Schon einige Jahre davor hatte Rita Franceschini (2006, 33), eine der herausragendsten Persönlichkeiten der europäischen Mehrsprachigkeitsforschung, angemerkt, dass das Thema „Mehrsprachigkeit“ - als Bezeichnung für unterschiedliche Formen individueller, sozialer und institutioneller Formen des Umgangs mit mehreren Sprachen - zentral in der sprachwissenschaftlichen Forschung geworden sei.

Die Allgegenwärtigkeit von Mehrsprachigkeit hatte aber bereits 1979 Mario Wandruszka in einem bemerkenswerten und zu Recht viel rezipierten Buch betont: „Der alte Satz ,Der Mensch ist das Wesen, das Sprache hat', ist eine ganz unzulängliche Bestimmung des Menschen. In Wahrheit muß [sic] er lauten: ,Der Mensch ist das Wesen, das mehrere Sprachen lernt ““ (Wandruszka 1979, 13). Aber obwohl Mehrsprachigkeit (auch) in Europa ein historisch stark verwurzeltes Phänomen ist, wurde diese auch von der (Sprach)Wissenschaft lange Zeit ignoriert - unter anderem aufgrund der romantischen Idee des Nationalstaates und der diesen mitbegründenden Vorstellung homoglossischer Gesellschaften. Letztere spiegelt sich auch in den vorherrschenden linguistischen Theorien bis weit ins 20. Jh. hinein wider, welche von getrennten, zählbaren und homogenen Sprachen ausgingen (vgl. Franceschini 2011, 345).

Franceschini $(2009,29)$ führt das vermehrte Interesse, aber vor allem die positivere Einstellung gegenüber dem Phänomen Mehrsprachigkeit von Seiten der Wissenschaft auf zwei Faktoren zurück; eine gesteigerte Sensibilisierung gegenüber Diversität, welche auch die sprachliche Homogenität in Frage stellt einerseits, die gesellschaftlichen Herausforderungen - auch sprachlicher Natur -, welche sich infolge der gesteigerten Mobilität und der Migrationsbewegungen seit der zweiten Hälfte des 20. und im 21. Jahrhundert ergaben, andererseits. Dass sich die Situation geändert hat und dass das Interesse für Mehrsprachigkeit in der wissenschaftlichen Community spürbar ist, zeigt sich 
unter anderem in der relativ hohen Anzahl an Einführungen und Handbüchern, die in den letzten 20 Jahren zum Thema erschienen sind. Es seien hier exemplarisch nur einige mit sprachwissenschaftlichem Hintergrund und Fokus genannt: Bilingualism: A Social Approach (Heller 2007), Handbook of Multilingualism and Multilingual Communication (Auer und Li 2007), Einführung in die Mehrsprachigkeitsforschung. Deutsch Französisch - Italienisch (Müller et al. [2006] 2011), The Routledge Handbook of Multilingualism (Martin-Jones, Blackledge und Creese 2012b), The Handbook of Bilingualism and Multilingualism (Bhatia und Ritchie [2004] 2013), Mehrsprachigkeit (Busch 2013), The Psycholinguistics of Bilingualism (Grosjean und Li 2013), Mehrsprachigkeit. Eine Einführung (Riehl 2014), The Handbook of Bilingual and Multilingual Education (Wright, Boun und García 2015), The Routledge Handbook of Migration and Language (Canagarajah 2017), Mehrsprachigkeit und Sprachenerwerb (Roche und Terrasi-Haufe 2018), The Routledge Handbook of Language and Superdiversity (Creese und Blackledge 2018), The Handbook of the Neuroscience of Multilingualism (Schwieter 2019), Handbuch Mehrsprachigkeits- und Mehrkulturalitätsdidaktik (Fäcke und Meißner 2019). Ganz zu schweigen von den zahlreichen Journals, aber auch Sammelbänden und Monographien, die den unterschiedlichsten Facetten der Mehrsprachigkeit gewidmet sind. Schon dieser kleine Überblick zeigt die Vielfältigkeit der Ansätze und Perspektiven, mit deren Hilfe versucht wird, sich dem Phänomen Mehrsprachigkeit anzunähern, auf, und offenbart zugleich die Notwendigkeit, diese zu ordnen und systematisch darzustellen.

Diese Breite an Ansätzen und Perspektiven zeigt sich aber nicht nur innerhalb der Linguistik mit ihren Teildisziplinen, sondern auch in vielen anderen sich der Thematik widmenden Disziplinen, von der Soziologie hin zur Kulturanthropologie und (Kultur)Soziologie ${ }^{4}$, der Literaturwissenschaft, der Fachdidaktik, der Medienwissenschaft ${ }^{5}$ und den Erziehungswissenschaften (vgl. Fürstenau 2011). So schreiben z.B. Dirim und Mecheril $(2010,99)$ :
Zwei- und Mehrsprachigkeit [...] wurde schon früh von der Erziehungswis- senschaft als pädagogische Fragestellung erkannt. [...] Nach einer Phase des relativen Stillstands in den 1980er-Jahren lässt sich in den letzten Jahren, vor allem ausgelöst durch die Ergebnisse der PISA-Studien, beobachten, dass die Erziehungswissenschaft sich in Kooperationen mit Sprachwissen- schaft und -didaktik des Sprach(en)themas annimmt.

Auch die Literaturwissenschaft ist immer mehr im Begriff, das lange vorherrschende Einsprachigkeitskonzept zu überwinden (vgl. Helmich 2016, 13). Literarische Mehrspra-

\footnotetext{
4 Vgl. z.B. Gerhards (2010).

5 Vgl. z.B. Bleichenbacher (2008).
} 
chigkeit rückt immer weiter ins Zentrum literaturwissenschaftlichen Interesses. Hat sich Bachtin zwar schon in den 1970er Jahren mit der Heteroglossie (vgl. Bachtin [1975] 1979) literarischer Produkte auseinandergesetzt - und damit einen der zentraleren Begriffe neuerer Mehrsprachigkeitsforschungsansätze vorgeschlagen -, so sind auch in der Literaturwissenschaft in den letzten zwanzig Jahren zahlreiche Publikationen mit Bezug zum Thema Mehrsprachigkeit entstanden. Es seien auch hier exemplarisch nur einige Titel - Handbücher, Monographien oder Sammelbände - genannt: Multilinguale Literatur im 20. Jahrhundert (Schmeling und Schmitz-Emans 2002), Plurilinguismo e letteratura (Brugnolo und Orioles 2002), Literatur und Vielsprachigkeit (Schmitz-Emans 2004), Exophonie. Anders-Sprachigkeit (in) der Literatur (Arndt, Naguschewski und Stockhammer 2007), In Babel's Shadow: Multilingual Literatures, Monolingual States (Lennon 2010), Literarische Mehrsprachigkeit. Sprachwechsel bei Elias Canetti und Ingeborg Bachmann (Radaelli 2011), Philologie und Mehrsprachigkeit (Dembeck und Mein 2014), Mehrsprachigkeit in der Literatur (Kremnitz [2004] 2015), Das literarische Leben der Mehrsprachigkeit. Methodische Erkundungen (Dembeck und Uhrmacher 2016), Ästhetik der Mehrsprachigkeit (Helmich 2016), Literarische Mehrsprachigkeit im österreichischen und slowenischen Kontext (Leben und Koron 2019), Literatur und Mehrsprachigkeit. Ein Handbuch (Dembeck und Parr [2017] 2020).

Nimmt man nur diese beiden Forschungsstränge vergleichend in den Blick, kristallisieren sich bereits unterschiedliche Konzeptionen von und vielfältige, teilweise (scheinbar?) diametral entgegengesetzte Perspektiven auf Mehrsprachigkeit und ihre Manifestationen heraus, die zeigen, wie komplex und facettenreich der Gegenstand ist und dass eine multiperspektivische, inter- und transdisziplinäre und auch weitere Disziplinen inkludierende Betrachtung zur Erfassung, Beschreibung und Analyse desselben notwendig ist.

\section{Zur Komplexität (der Erfassung) mehrsprachiger Welt(en)}

Phänomene wie die schon erwähnte Globalisierung und die weltweit gesteigerte Mobilität in ihren unterschiedlichen Formen ${ }^{6}$ sowie die Entwicklung neuer Technologien und die Verbreitung sozialer Medien tragen zu einer wachsenden gesellschaftlichen und sprachlichen Vielfalt und mitunter zu einer gesteigerten Wahrnehmung dieser Vielfalt bei. Unter anderem hat die gesteigerte Aufmerksamkeit für die Sprachrechte minoritärer

\footnotetext{
${ }^{6}$ Neben transkontinentalen Flucht- und Migrationsbewegungen sind z.B. auch Formen der (temporären) innereuropäischen Arbeitsmigration, man denke an Erntehelfer*innen oder an Akteur*innen in der $\mathrm{Ca}$ re-Arbeit, sowie Formen der "Elitenmigration' (Stichwort: Expats) zu verzeichnen. Letztere haben erleichterten Zugang zum „cosmopolitan capital“ (Garrido 2017,361), und daher bessere Chancen, daraus symbolisches und/oder ökonomisches Kapital zu gewinnen.
} 
Sprachgemeinschaften teilweise zu einer (relativen) Aufwertung des sprachlichen Potenzials von Mehrsprachigen beigetragen. ${ }^{7}$ Die Transformationen der globalisierten Weltökonomie haben Sprache in vielen Fällen aber auch zu einer commodity ${ }^{8}$ gemacht, zu einer Ware, welche dem wirtschaftlichen Profit zu dienen hat. Sprache ist in dieser Hinsicht „not only an integral, if not the only, part of the work process; it is also frequently the work product" (Heller 2010a, 350). Die Auffassung von Sprache als Gut, als intellektuelles (und symbolisches) Kapital, hat weitreichende direkte und indirekte Folgen. Sie trägt dazu bei, Sprachhierarchisierungen zu festigen und zu perpetuieren. Auf der einen Seite stehen die ,guten', richtigen', auf dem globalen Markt, verwertbaren' Sprachen, die ,Weltsprachen` wie Englisch, aber auch Russisch oder Chinesisch, deren Erwerb angestrebt wird, und die „einen Ausdruck der staunenden Bewunderung für die Sprachkompetenz der Sprecher_innen bei den Zuhörenden hervorrufen“, auf der anderen Seite finden sich die zu vernachlässigenden, ,falschen` bzw. ,schlechten` Sprachen, die Minderheiten- und Migrantensprachen, die "gesellschaftlich delegitimierten Sprachen" (Heinemann und Dirim 2016, 199).9 Dies hat die Unterscheidung zwischen einer ,nützlichen', anzustrebenden und zur Schau getragenen Elitemehrsprachigkeit und einer unsichtbar gemachten bis stigmatisierten Armutsmehrsprachigkeit zur Folge und fördert offenen oder verdeckten Linguizismus ${ }^{10}$ (vgl. Dirim 2010; Springsits 2015). Aber auch kleinere, lokale Sprachen werden der Kommerzialisierung unterworfen und dienen in bestimmten (Nischen-)Märkten als Zeichen von Authentizität, Nähe und Regionalität ${ }^{11}$ (vgl. Heller 2010a; Duchêne und Heller 2012; Barakos und Sellek 2019, 8). Die linguistic landscapes werden Schauplatz für den Wettbewerb unterschiedlicher Ak-

7 Dies ist aber nicht immer der Fall. Die Aufwertung sprachlicher Rechte einer minoritären Gruppe kann durchaus bedeuten, dass diese wiederum in der Homogenitätslüge gefangen ist und sich selbst konterkarierend Einsprachigkeitsideale pflegt und sich sprachlich (und demzufolge sozial und wirtschaftlich) von der majoritären Gruppe absondern möchte (dazu vgl. z.B. Peterlini 2019). Minoritäre Gruppen sind auch nicht per se minderheitenfreundlich - häufig gestehen sie die für sich erkämpften Sprachrechte anderen minoritären Gruppen nicht zu. Dies kann wiederum zu einer Art forcierter Selbstethnisierung der diskriminierten (Sprach-)Gruppe(n) führen.

8 Zum Konzept der Kommodifizierung von Sprache vgl. Heller (2010b); kritisch dazu Block (2019).

9 Aus liberalismuskritischer Perspektive werden daher auch wissenschaftlich-politische Ansätze wie language-as-ressource (vgl. dazu Ruiz 2010), welche die Vorteile von Sprachenvielfalt und von multiplen Sprachkenntnissen hervorheben, in Frage gestellt, da die Betrachtung von Sprache als Ressource Gefahr laufe, in einen ökonomistischen Diskurs zu münden und zentrale Fragen wie die der sprachlichen Rechte als allgemeine Menschenrechte einer Kosten-Nutzen-Perspektive zu unterstellen (vgl. Grin 1996, 2005).

${ }^{10}$ Als „Neo-Linguizismus“ definiert Dirim (2010, 95-96) verdeckte - aber deswegen nicht weniger verheerende - Formen des Linguizismus, die scheinbar ,kompatibler` mit demokratischen Systemen sind, welche offene Formen der Diskriminierung nicht dulden.

${ }^{11}$ Man denke z.B. an die Verwendung von Dialekten in der Werbung international agierender Unternehmen oder an Fremdenverkehrsangebote, in denen der Dialektgebrauch von Seiten der Ortsansässigen sozusagen ,im touristischen Paket' mit inbegriffen ist. Für ein Beispiel aus Kärnten vgl. Omann (2020). 
teur*innen um die Visibilität - und damit die Legitimierung - unterschiedlicher Sprachen, um die sprachliche Gestaltung und Aneignung des öffentlichen Raums. Spracheinstellungen und -ideologien haben Sprachregimes ${ }^{12}$ zur Folge, welche als „a set of constraints on individual language choices" (Coulmas 2005,7) bestimmen, welche Sprache(n) und sprachliche Praktiken legitim sind, welche dagegen als unerwünscht abgestempelt oder ausgeblendet werden.

Die Kommerzialisierung von Sprache hat aber noch weitere Folgen. Mehrsprachige Sprachqualifikationen, die nach standardisierten Methoden und Tests zertifiziert werden, werden immer unerlässlicher für die beruflichen Karrierewege. Vor diesem Hintergrund hat sich eine profitable Sprachindustrie entwickelt, die von ,klassischen' Sprachkursen (und des „Edutourismus)“, Übersetzungs- und Dolmetschservices sowie der Entwicklung von „objektiven“ Tests und Sprachlernmaterialien über bi- oder mehrsprachige Schulen bis hin zu den in den letzten Jahren immer elaborierter und effizienter gestalteten Programmen und Apps zur automatischen Spracherkennung und Übersetzung reicht. Aber auch die Sprachwissenschaft sieht sich vor neuen Herausforderungen und versucht, diesen adäquat zu begegnen (vgl. Lüdi 2011).

All diese Entwicklungen können wie oben bereits angedeutet nur in einem multi-, inter- und transdisziplinären Rahmen ihrer Komplexität angemessen und multiperspektivisch untersucht werden - und sie verlangen auch nach einem Umdenken in der Beschäftigung mit und in der Konzeptualisierung von Sprache.

\section{Viele oder doch nur eine Sprache?}

In den letzten Jahrzehnten hat sich in Teilen der sprachwissenschaftlichen sowie didaktischen und pädagogischen Forschung zur Mehrsprachigkeit ein Perspektivwechsel vollzogen. Die Vorstellung von diskreten, voneinander getrennten Sprachsystemen, die in weiten Teilen der Gesellschaft, aber auch der Wissenschaft lange herrschte, wurde zugunsten der Idee komplexer sprachlicher Ressourcen aufgegeben. Dabei wird implizit auf eine Unterscheidung der Struktur der Sprache in Ebenen rekurriert, die u.a. vom Romanisten Eugeniu Coseriu schon im Jahre 1980 vorgeschlagen worden war, und zwar:

a) die universelle Ebene des Sprechens im allgemeinen [sic] ohne jegliche historische Bestimmung (das, was man auch „Sprache im allgemeinen“ oder „langage“ nennen könnte);

b) die historische Ebene der Sprachen (im Plural), d.h. der historisch gewordenen gemeinschaftlichen Traditionen des Sprechens; und

\footnotetext{
${ }^{12}$ Dazu vgl. u.a. Costa (2019), Irvine (2019).
} 
c) die individuelle Ebene der Texte (Coseriu [1980] 1988, 47)

Obwohl es dem Tübinger Sprachwissenschafter mit dieser Unterscheidung in primis darum ging, die Unterscheidung zwischen Sprache und Dialekt als eine kontingent-historische fass- und definierbar zu machen, um damit das Varietätengefüge einer historischen Sprache - die er als eine komplexe und aus unterschiedlichen Varietäten bestehende Architektur betrachtete - beschreiben zu können, erweist sich diese Unterscheidung auch als nützlich, wenn man zwischen der historisch-kulturellen Ebene der Einzelsprache und der individuell-menschlichen Ebene unterscheiden möchte, wie dies in neueren Ansätzen zur Mehrsprachigkeitsforschung getan wird, die einerseits aus didaktisch-pädagogischer Perspektive, andererseits aus neurolinguistischer Sicht kommen.

\subsection{Viele Sprachen, eine Sprachverarbeitung}

Neurolinguistische Untersuchungen haben in den letzten zwanzig Jahren gezeigt, dass Sprachen im Gehirn als komplexes, aber einheitliches System verarbeitet werden: „the brains of individuals speaking more than one language is considered as a complex and unique system rather than the sum of monolingual brains" (Piccardo und Aden 2014, 241). Gingen Forscher*innen noch vor nicht allzu langer Zeit von getrennten neuronalen Netzwerken aus, wenn bilinguale Individuen zwei (oder mehrere) Sprachen nicht simultan erlernen (vgl. Bickes 2004; Bickes und Pauli 2009, 93-94), haben Untersuchungen an der Freien Universität (vgl. Videsott et al. 2010) in Bozen/Brixen gezeigt, dass die Sprachverarbeitung zwar getrennt nach Sprachbereichen (Phonologie, Morphologie, Syntax, usw.) erfolgt, jedoch nicht nach historischen Sprachen - und dies unabhängig von Erwerbsalter und -modalitäten:

Auf kognitiver Ebene scheint es hingegen die extralinguistisch unterschiedenen 'Einzelsprachen' nicht zu geben: Vielmehr scheint 'eine' Sprache bzw. Sprachfähigkeit vorhanden zu sein, die sich in den verschiedenen Sprachebenen Phonetik, Morphologie, Syntax, Semantik, Lexikon usw. [...] manifestiert, und die erst nachträglich von den historischen Einzelsprachen 'modelliert' wird. (Videsott 2011, 208; vgl. auch Videsott 2020, 476)

Andere, wie z.B. Schneider (2015, 239), relativieren zumindest die Bedeutung des Erwerbsalters und sehen einen anderen Faktor als ausschlaggebend, und zwar den höheren Verarbeitungsaufwand, der nötig ist, um Strukturen einer Sprache abzurufen, wenn diese im geringen Maße beherrscht wird und betonen, dass „Spätbilinguale mit hohen Kompetenzen in der Zweitsprache [...] nicht unbedingt unterschiedliche neuronale Repräsentationen der beiden Sprachen auf[weisen]“. 
Diesen Forschungen gemeinsam ist die Erkenntnis, dass keine neuronale Trennung bei der Verarbeitung von unterschiedlichen Sprachen im Gehirn nachzuweisen ist - im Gegenteil, sie werden gemeinsam abgespeichert und, wenn abgerufen, aktivieren dieselben Gehirnareale und neuronalen Netze. Die Unterscheidung zwischen Sprachen als diskreten Systemen, die spätestens seit dem Strukturalismus vorherrschend ist, ist also keine, die im Individuum zu finden ist, sondern eine historisch-kulturelle. So betrachtet Franceschini $(2011,350)$ multilinguale Sprachpraktiken als nichts Anderes als einen Fall von sprachlicher Variation im Gebrauch: „multilingualism is only a special case of variable use, languages being separated ideologically, but not psycholinguistically" (vgl. auch das Konzept der „multicompetence“ wie ihn Hall, Cheng und Carlson 2006 vorschlagen).

\subsection{Komplexes Potential, eine Ressource}

Dass Sprache (d.h. die Einzelsprache) ein soziales und historisches Konstrukt ist, wurde vor allem in der sogenannten Debatte um named languages (vgl. unter anderen Makoni und Pennycook 2007; Heller 2007; Otheguy, García und Reid 2015) wieder aufgegriffen, bei der betont wird, dass die Vorstellung von getrennten, zählbaren, diskreten Sprachsystemen bei mehrsprachigen Individuen eine sozial-historisch und ideologisch begründete Außensicht ist, welche dem sprachlichen Repertoire und den sprachlichen Praktiken multilingualer Menschen nicht entspricht. Diese sind durch den Rückgriff auf alle vorhandenen sprachlichen Ressourcen charakterisiert, bei denen weder Grenzen zwischen einzelnen Sprachsystemen noch zwischen unterschiedlichen diaphasischen, diastratischen oder diatopischen Varietäten einer historischen Sprachen existieren, sondern einfach eine Sprachlichkeit. Darauf aufbauend und in die gleiche Kerbe schlagend sind neuere Forschungsansätze, die aus pädagogischer und (fremd-)(sprachen-)didaktischer Perspektive kommen, und die unter dem Terminus Translanguaging subsumiert werden können. ${ }^{13}$ Damit werden sprachliche Praxen mehrsprachiger Individuen beschrieben und pädagogische bzw. didaktische Ansätze, die diesen gerechter werden möchten:

$[\mathrm{T}]$ ranslanguaging is an approach to the use of language, bilingualism and the education of bilinguals that considers the language practices of bilinguals not as two autonomous language systems as has been traditionally the case, but as one linguistic repertoire with features that have been societally constructed as belonging to two separate languages. (García und Li2014,2)

Translanguaging-Ansätze vollziehen damit einen Wechsel von einer (aus einem einzelsprachlichen Systemgesichtspunkt) defizit-orientierten Perspektive hin zur Aufwertung

\footnotetext{
${ }^{13}$ Andere Termini, die in der Literatur verwendet werden, um denselben bzw. ähnliche Ansätze beschreiben, sind u.a. Polylanguaging, Metrolanguaging, language meshing.
} 
komplexer kommunikativer Potenziale und Ressourcen; sie betrachten Phänomene wie Code-Mixing oder Code-Switching nicht als Vermischungen oder Verwechslungen von ,starren ' Codes, sondern als Formen des „,discursive multilingualism““ (Franceschini 2011, 346), als diskursiv kompetenten und kommunikativ erfolgreichen Rückgriff auf alle im eigenen, komplexen aber einheitlichen Repertoire vorhandenen Ressourcen. Es handelt sich um eine epistemologische Wende, welche die Blickrichtung weg von der Betrachtung der Sprache als von den Sprecher*innen und den Kommunikationssituationen losgelöst funktionierendes System und hin zu einer Aufwertung der individuellen und konkreten Ressourcen und kommunikativen Strategien richtet. Streng genommen wird daher nicht von mehreren Sprachen - und daher auch nicht von mehrsprachigen Individuen gesprochen, sondern von komplexen sprachlich-kommunikativen Repertoires, von den „real bits and chunks of language that make up a repertoire, and of real ways of using this repertoire in communication" (Blommaert 2010, 173).

Der Ausgangspunkt von Translanguaging-Ansätzen sind also die konkreten alltäglichen kommunikativen Praktiken, die individuellen Akte des Sprechens. ${ }^{14}$ Sie stellen das Individuum mit seiner (Sprach-)Biographie ${ }^{15}$ ins Zentrum, mit dem Ziel, die agency der Subjekte zu erhöhen und pluralistische Ansätze zu fördern. Mehrsprachigkeit wird hier als dynamische kommunikative Kompetenz aufgefasst. Ein zentraler Kritikpunkt aus dem Umfeld von Translanguaging-Vertreter*innen richtet sich gegen das Konzept der Standard- bzw. Referenzsprachen, das als ideologisches Konstrukt, als starres Gebilde und als potentielle Grundlage für Linguizismus und Sprachhierarchisierungen betrachtet wird, so wie dies auch in Ansätzen des „Inclusive Multilingualism“ (Backus et al. 2013) der Fall ist. Translanguaging-Konzepte möchten (gesellschaftlich) erfolgreiche, aber höchst umstrittene Vorstellungen wie die des Semilingualismus ${ }^{16}$ oder von „partial or limited bilinguals“ (Cummins 1981,39) überwinden und u.a. die Förderung der language awareness sowohl auf struktureller als auch auf emotionaler, individueller, sozialer und machtbezogener Ebene fördern.

\subsection{Die Bedeutung von Namen}

Wenn es also zwar auf neurokognitiver und diskursiver Ebene nicht möglich scheint, von getrennten Sprachen zu sprechen und das Konzept der Einzelsprache in Teilen der Wissenschaft kritisch hinterfragt wird, so spielt die Vorstellung von ,abgrenzbaren', ,zählbaren' named languages als Kulturprodukten doch eine sehr grundlegende Rolle für die

\footnotetext{
${ }^{14}$ Zur Kritik an Translanguaging-Konzepten vgl. u.a. Mac Swan (2017).

${ }^{15}$ Dazu vgl. u.a. Gombos (2019).

${ }^{16}$ Eine kritische Rekonstruktion des Erfolges dieses Konzeptes findet sich in Salö und Karlander (2018).
} 
Selbstdefinition von Individuen und die Gesellschaft. ${ }^{17}$ Historische Sprachen sind zwar kontingent, sie existieren aber als soziale Konstrukte und bedingen Vorstellungen über Sprache(n), Sprachideologien und sprachliches Handeln von Sprecher*innen: „Languages are inventions and ideological constructs, but they are simultaneously perceived as natural and experienced as real by most people“ (Ritzau 2015, 663). Dies zeigt sich auch am Gebrauch von ,Sprachetiketten` wie Muttersprache, Fremdsprache, Zweitsprache, Amtssprache, Minderheitensprache u.ä., die das individuelle, gesellschaftliche und institutionelle Bedürfnis, Sprachen zu unterscheiden und je nach (emotionaler) Bedeutung und Funktion(en) für das einzelne Individuum oder für eine bestimmte Gesellschaft zu kategorisieren (vgl. Aronin, Ó Laoire und Singleton 2011; zu native speakers vgl. Khakpour 2016), widerspiegeln. Solche Bezeichnungen, die Sprachen teils bottom-up, teils top-down und teils in wechselseitiger Beziehung dieser zwei Richtungen zugewiesen werden (vgl. Aronin, Ó Laoire und Singleton 2011, 172), reflektieren die Einstellungen und die Bindungen zu sowie das Ansehen von den jeweils betroffenen historischen Einzelsprachen und tragen daher stark zu Etablierung von Sprachregimes bei. Während daher manche Autor*innen wie Makony und Pennycook (2007) und Pennycook (2010) für die Aufgabe dieses Konstruktes plädieren, schlagen andere dessen Beibehaltung, aber eine weitere Umkehrung der Perspektive, welche den scheinbaren Widerspruch auflösen könnte, vor. Es sei hier nur der von Turner und Lin (2017) vorgestellte integrative und für die Überwindung der aufgezeigten Widersprüchlichkeit in pädagogischen Kontexten angedachte Ansatz erwähnt, in dem das Ziel einer Translanguaging-basierten Pädagogik immer noch der Erwerb einer bestimmten, als getrennt konzipierten (Fremd-)Sprache ist. Sie schlagen vor, ,the educational objective from named language to linguistic repertoire“ (Turner und Li 2017, 2) zu verschieben. Der Perspektivenwechsel vom Erlernen einer einzelsprachlichen Norm hin zur Erweiterung und zum Aufbau kommunikativer Ressourcen durch eine (neue) Sprache würde auch zur Dekonstruktion von Sprachhierarchien und von darauf beruhenden Stigmatisierungs- und Diskriminierungserfahrungen beitragen. Einen ähnlichen Perspektivwechsel auch jenseits rein sprachdidaktischer Kontexte auf breiter gesellschaftlicher Ebene zu erreichen, würde bedeuten, die Grenzen geschlossener Sprecher*innengemeinschaften zugunsten offener transnationaler Kommunikationsgemeinschaften aufzugeben und Linguizismus, Sprachhierarchisierung und sprachliche Ausgrenzung dezidiert zu bekämpfen. Dabei ist jedoch eine multiperspektivische inter- und transdisziplinäre Beschäftigung mit der Mehrsprachigkeit einerseits und mit den Diskursen darüber andererseits notwendig, um sowohl linguistische, aber auch so-

${ }^{17}$ Dies ist den Vertreter*innen von Translanguaging-Ansätzen durchaus bewusst, vgl. z.B. Otheguy, García und Reid (2015, 293), Cummins (2017, 414), Paulsrud et al. (2017, 15), Otheguy, García und Reid (2019, 628), Carbonara und Scibetta $(2020,4)$. 
ziopolitische, sprachenrechtliche, psychologische, didaktische, pädagogische und literarische bzw. literaturhistorische Aspekte angemessen einzubinden.

\section{Mehrsprachigkeitsforschung an der Alpen-Adria-Universität}

Mehrsprachigkeitsforschung blickt an der noch relativ jungen Alpen-Adria-Universität auf eine bemerkenswert lange Tradition zurück und ist fest im Forschungsprofil der Universität verankert. Der Alpen-Adria-Universität kommt in Österreich sicherlich - und sicherlich nicht zufällig - eine Vorreiterrolle in Bezug auf die Auseinandersetzung mit den unterschiedlichsten Gesichtspunkten der Mehrsprachigkeit zu. Diese erfolgt im Rahmen von regionalen, nationalen und grenzüberschreitenden Projekten und weist im Sinne einer angewandten Wissenschaftsprofilierung einen starken Fokus auf sowohl wissenschaftlich als auch gesellschaftspolitisch relevante Aspekte auf. Dass Mehrsprachigkeitsforschung an der Alpen-Adria-Universität in Klagenfurt, am „Schnittpunkt dreier Kulturen " gelegen, wie in der Selbstbeschreibung der Universität betont wird ${ }^{18}$, eine große Rolle spielt, zeigen die Aktivitäten der dort angesiedelten, inter- und transdisziplinären $A G$ Mehrsprachigkeit. Es ist auch kein Zufall, dass im Jahre 2001 - dem europäischen Jahr der Sprachen - gerade in Klagenfurt die Vertreter ${ }^{*}$ innen des österreichischen Verbands für Angewandte Sprachwissenschaft (VERBAL) eine Erklärung verabschiedeten die Klagenfurter Erklärung zur österreichischen Sprachenpolitik -, in der zahlreiche wichtige Forderungen zur Entwicklung einer mehrsprachigen Sprachstrategie in Österreich formuliert wurden, welche die Förderung der individuellen und gesellschaftlichen Sprachrechte sowie der individuellen und gesellschaftlichen Mehrsprachigkeit und der Visibilität derselben in der Öffentlichkeit zum Ziel hatten. Eine weitsichtige Erklärung, die zehn Jahre später - in einer überarbeiteten Fassung und stärker im Kontext europäischer Rahmenstrategien und Zielsetzungen eingebettet - wieder veröffentlicht wurde, und die auch heute, fast zwanzig Jahre später, immer noch höchst aktuell ist. ${ }^{19}$ Erneut in Klagenfurt wurden im Jahre 2008 die 15 Thesen zur Mehrsprachigkeit (Wintersteiner, Gombos und Gronold 2008) verfasst, welche die Förderung und positive Akzeptanz der unterschiedlichen Manifestationen der Mehrsprachigkeit zu einem erstrebenswerten Ziel gesellschaftlicher und bildungspolitischer Bemühungen erklären.

\footnotetext{
${ }^{18}$ Vgl. das Profil auf der Homepage der Universität unter https://www.aau.at/universitaet/profil/, aber auch die Selbstbeschreibung des Instituts für Germanistik unter https://www.aau.at/germanistik/ oder des International Office unter https://www.aau.at/universitaet/organisation/administrationverwaltung/international-office/ [Stand: 30.09.2020].

${ }^{19}$ Vgl. https://www.univie.ac.at/linguistics/verbal/fileadmin/user_upload/Stellungnahmen/ST_2011_ Klagenfurter_Erklaerung_Revisited_FIN.pdf [Stand: 30.09.2020].
} 
Aber Mehrsprachigkeit hat auch in der Lehre einen festen Platz. Es ist an dieser Stelle nicht möglich, das vielseitige Angebot an Lehrveranstaltungen zum Thema, das zahlreiche Lehrende im Rahmen der unterschiedlichen Studien anbieten, darzustellen. Hervorzuheben sind aber sicherlich das Wahlfachmodul Mehrsprachigkeit interdisziplinär sowie die gleichnamige, seit über zehn Jahren jährlich stattfindende Ringvorlesung, welche Studierenden aller Fächer das Thema Mehrsprachigkeit aus linguistischer, sozio-kultureller, literatur- und medienwissenschaftlicher, psychologischer, didaktischer und pädagogischer Perspektive näherbringt und die bei den Studierenden auf reges Interesse stößt. Darüber hinaus haben die Hochschulen im Entwicklungsverbund Südost ${ }^{20}$ im Rahmen der PädagogInnenbildung neu die interdisziplinäre Beschäftigung mit dem Thema Mehrsprachigkeit als festen Bestandteil in das Mastercurriculum aller Lehramtsstudien aufgenommen - im Bewusstsein, dass gerade die Schule als Spiegelbild der Gesellschaft im Spannungsfeld zwischen monolingualem Habitus (Gogolin 1994) und sprachlicher Vielfalt von den künftigen Lehrkräften einen offenen, bewussten Umgang mit den sich stellenden Herausforderungen und bietenden Chancen verlangt. Die Anbindung der 2018 geschaffenen Professur für Mehrsprachigkeitsforschung an die institutsübergreifende und interinstitutionell agierende School of Education spiegelt die Notwendigkeit der Beschäftigung mit Mehrsprachigkeitsfragen im Bereich der Professionalisierung von Lehrkräften wider und begünstigt den interdisziplinären und fachübergreifenden Austausch und den Kontakt mit der Praxis.

Universitäre Lehre und Forschung sollten die Mehrsprachigkeitsphänomene in ihrer Wechselbeziehung mit deren subjektiver und kollektiver Wahrnehmung sowie gesellschaftlichen und politischen Diskursen zur Mehrsprachigkeit in den Blick nehmen. Dies führt weg von kanonisierten Wissensbeständen und unter anderem hin zur Erforschung und Beforschung des subjektiven Erlebens von Mehrsprachigkeit, zur parallelen und verknüpften kritischen Analyse des Gegenstands Mehrsprachigkeit einerseits und der gesellschaftlichen und wissenschaftlichen Diskurse über diese andererseits und zur Untersuchung der Wechselwirkungen zwischen diesen Ebenen. Ein trans- und interdisziplinäres Fach Mehrsprachigkeitsforschung kann kein selbstverständlicher und einfacher Weg sein, denn es verlangt nach der Sprengung verankerter disziplinärer Grenzen und kann nur in einer fruchtbaren Verbindung von Forschung und Lehre erfolgen. Die akademisch-institutionelle Verortung der genannten Professur für Mehrsprachigkeitsforschung am Institut für Kulturanalyse bietet ideale Voraussetzungen dafür, dass

\footnotetext{
${ }^{20}$ Dem neben der AAU die Karl-Franzens-Universität Graz, die Kirchliche Pädagogische Hochschule Graz, die Pädagogische Hochschule Burgenland, die Pädagogische Hochschule Kärnten, die Pädagogische Hochschule Steiermark, die Universität für Musik und darstellende Kunst Graz sowie die Technische Universität Graz angehören.
} 
„de[r] sprechende[.] Mensch[.] bzw. das multilinguale Subjekt in den Mittelpunkt“ gestellt, „die soziale und diskursive Konstruiertheit sprachlicher Kategorien“ herausgearbeitet und „sprachliche Praktiken in unterschiedlichen sozialen Kontexten in den Blick“ (Busch 2013, 7) genommen werden kann. Langfristig scheint die Vorstellung der Etablierung der Mehrsprachigkeitsforschung als universitäres Fach - wie sie teilweise an einigen Universitäten im deutschsprachigen Raum (und nicht nur) beobachtbar ist ${ }^{21}$ eine Möglichkeit zu sein, die inter- und transdisziplinäre Betrachtung der Mehrsprachigkeit als Querschnittsmaterie weiter zu etablieren und der wissenschaftlichen Erforschung und der akademischen Lehre in diesem Bereich den ihrer gesellschaftlichen Relevanz entsprechenden Platz einzuräumen.

Die hier kurz umrissene Bandbreite der an der Alpen-Adria-Universität betriebenen Forschung zum Thema Mehrsprachigkeit exemplarisch aufzuzeigen, versuchte schon der von Allan James im Jahre 2003 herausgegebenen Band Vielerlei Zungen (James 2003): Die gesammelten Beiträge stammten aus der Linguistik, der Spracherwerbsforschung, der Erziehungswissenschaft, der Literaturwissenschaft, der Psychologie und der Medienwissenschaft. Das bereits damals breite Spektrum an Disziplinen sieht sich heute mindestens um die Sprachdidaktik sowie die unterschiedlichen Fachdidaktiken, Geschichte, Kulturanthropologie, Gebärdensprachenforschung usw. erweitert. Die vorliegende Ausgabe von Colloquium: New Philologies möchte daran anknüpfen und somit auch der Beschreibung der veränderten Bedingungen und Formen von Mehrsprachigkeit auf der Grundlage neuer methodologischer und theoretischer Ansätze einen adäquaten Rahmen bieten.

\section{Die Beiträge in dieser Ausgabe}

Ebenso vielfältig wie die inter- und transdisziplinären Forschungsansätze sind die Beiträge, die in dieser Ausgabe von Colloquium: New Philologies gesammelt sind. Der Beitrag von Allan James Various Tongues Updated: Mediatisation, Visualisation and the Digitalisation of Social Multilingualism - the Continuing Role of English [25-40] stellt die ideale Brücke zu der mehrfach zitierten Publikation Vielerlei Zungen (James 2003) dar und bietet eine Überprüfung der damals gezeichneten Perspektiven und zugleich ein Update bezüglich neuerer Entwicklungen in der sprachwissenschaftlichen Mehrsprachig-

\footnotetext{
${ }^{21}$ Man denke z.B. an den Masterstudiengang „Empirische Mehrsprachigkeitsforschung“, der gemeinsam von der TU Dortmund und der Ruhr-Universität Bochum angeboten wird, an den Master „Mehrsprachigkeit und Regionalität“ der Universität Regensburg oder an den Master „Mehrsprachigkeitsforschung“ an der Universität Freiburg/Université Fribourg; aber auch das Kolloquium Mehrsprachigkeit an der Universität Innsbruck kann als Zeichen der Institutionalisierung des interdisziplinären wissenschaftlichen Diskurs zu mehrsprachigkeitsbezogenen Themen angesehen werden.
} 
keitsforschung. Besondere Aufmerksamkeit erhält dabei der Perspektivenwechsel, der, wie schon erwähnt, von Code-Switching zu Translanguaging-Ansätzen erfolgt ist. Die Medialisierung und Visualisierung von Sprache und die Hybridisierungsphänomene, die diese kennzeichnen, werden unter die Lupe genommen; unter besonderer Berücksichtigung von Sprachhierarchisierungen, die sich in deren Präsenz in der linguistic landscape manifestieren - mit der Dominanz von Anglizismen bzw. Xenoanglizismen ${ }^{22}$ in nicht (primär) anglophoner Kontexten, um schließlich einige Beispielanalysen von hybriden Sprachpraktiken in sozialen Medien näher zu illustrieren.

Magdalena Kaltseis und Ursula Doleschal präsentieren in ihrem Beitrag Präferenzen, Interesse(n) und Gründe von SchülerInnen für das Erlernen von (Fremd-)Sprachen. Eine Pilotstudie an Kärntner Schulen [41-70] die bemerkenswerten Ergebnisse einer ersten Studie, welche das Interesse von Schüler*innen in Kärnten an Fremdsprachen, die in ihrer Schule nicht angeboten werden und ihre Bereitschaft, diese zu lernen, erhoben hat. Die Vielfalt der genannten Sprachen, aber vor allem die relativ hohe Anzahl an Kindern, welche $\mathrm{B} / \mathrm{K} / \mathrm{S} /$ und Russisch angeben - auch bei den lebensweltlich monolingual deutschsprechenden Kindern - zeigen, dass das Fremdsprachenunterrichtsangebot, welches sich auf (vermeintlich?), marktfähige‘ Sprachen fokussiert, mit der Förderung einer (vermeintlichen) Elitemehrsprachigkeit (vgl. De Costa 2019) weder die gesellschaftliche Sprachenvielfalt abbildet noch den kommunikativen Bedürfnissen der Schüler*innen gerecht wird. Im Gegenteil: Diese Angebote fixieren und perpetuieren Hierarchisierungen von Sprachen (und deren Sprecher*innen) und können somit, direkt oder indirekt, Formen der Diskriminierung und des Linguizismus fördern.

Isabella Matticchios Le metriche ritmiche applicate allo studio del parlato bilingue. Stato dell'arte e implicazioni perpossibili studi nell'Alpe Adria [71-104] stellt einen informierten und kritischen Forschungsbericht über Studien dar, die methodologisch unter Rückgriff auf das Konzept der rhythm metrics für die instrumentelle Messung von Rhythmusintervallen erstellt worden sind. Nachdem sie die Vorteile und Probleme dieses theoretischen Ansatzes kritisch diskutiert hat, fokussiert sie sich auf solche Studien, die sich mit dem (Erwerb des) Sprechrhythmus(') bei bi- bzw. multilingualen Sprecher*innen beschäftigen - insbesondere mit Fällen von Sprachenkombinationen, in denen eine der betroffenen Sprache silben-, die andere akzentzählend ist. Sie unterscheidet dabei zwischen Studien, welche Fremdsprachenlerner*innen als Proband*innen hatten, und Studien, die dagegen Sprecher*innen untersuchen, welche zwei oder mehrere Sprachen simultan erworben haben. Nach dieser kritischen Darstellung zeigt sie weitere Forschungsperspektiven im mehrsprachigen Alpen-Adria-Raum auf und sie betont insbesondere die Notwendig-

\footnotetext{
${ }^{22}$ In Anlehnung an das von Vedovelli (2019) vorgeschlagene „xenoitalianismi“ für italienisch anmutende Elemente, die in nicht zentral italienischsprachigen Kontexten entstehen.
} 
keit von Studien, die Rhythmusstrukturen bei bilingualen Slowenisch-Italienisch- bzw. Italienisch-Slowenisch-Sprecher*innen analysieren.

Gerd Hentschel und Tilmann Reuther stellen in ihrem Beitrag Ukrainisch-russisches und russisch-ukrainisches Code-Mixing. Untersuchungen in drei Regionen im Süden der Ukraine. Ein dreijähriges Forschungsprojekt im Rabmen des D-A-CH-Programms von FWF und DFG [105-132] ihr Forschungsvorhaben vor, das im Rahmen eines binationalen Forschungsprojekts zum Suržyk an der Universität Oldenburg(D) und an der AAU umgesetzt wird. Beim Suržyk handelt es sich um eine aus dem Kontakt zwischen Ukrainisch und Russisch entstandene Hybridvarietät, die in der informellen Kommunikation - sowohl in der Familie als auch in der Gesellschaft - in der Ukraine verwendet wird, wobei die Autor*innen von zwei Varietäten desselben, einem „alten“ und einem „,neuen Suržyk“ ausgehen. In ihrem Beitrag präsentieren sie die Forschungslage zum Thema, um dann den historisch-politischen Hintergrund zu beleuchten, vor dem diese Sprache entstanden ist und gesprochen wird, sowie die Verortung des Suržyk im linguistischen Paradigma, um dann Forschungsmethode und Analyseverfahren darzustellen.

Gerhard Katschnig nähert sich aus kulturwissenschaftlicher Sicht der historischen Mehrsprachigkeit am Beispiel von Hermann von Carinthia. Der Philosoph, Mathematiker und Astronom nahm eine zentrale Stellung im kulturellen und wissenschaftlichen Leben seiner Zeit ein. Als Übersetzer wurde er durch seine Translation astronomischer und naturwissenschaftlicher Texte aus dem Arabischen ins Lateinische zu einer der Hauptfiguren für den fruchtbaren arabisch-westlichen Kulturtransfer. Seine tiefgehende Auseinandersetzung mit der arabischen Sprache und Kultur machte ihn zu einem der wichtigsten Botschafter arabischer Kultur im Europa des 12. Jahrhunderts. Übersetzung und Mehrsprachigkeit im 12. Jahrhundert: Hermann von Karinthia [133-153] beleuchtet die Bedeutung des translatorischen Wirkens dieses wichtigen und doch noch zum Teil unerforschten Gelehrten.

Andreas Hudelists Aesthetic multilingualism as ontological relativism. Milorad Pavic's Dictionary of the Khazars and Mark Z. Danielewski's House of Leaves [154-167] eröffnet eine stärker literarisch orientierte Beschäftigung mit Mehrsprachigkeit. Unter ,ästhetischer Mehrsprachigkeit' versteht der Autor die Fähigkeit, die eigene Umwelt in mehr als einer Erfahrungsform, in mehr als einem Wahrnehmungsmodus wahrzunehmen. Dies bezieht sich insbesondere auf die Rezeption kultureller Werke, die in prozessualem Dia$\log$ zwischen Autor und Rezipient ${ }^{*}$ innen erfolgt. Offene Kunstwerke, wie der ,Lexikonroman' Dictionary of the Khazars (orig, Hazarskirečnik, 1984) des serbischen Autors Milorad Pavić und der 2000 als Buch erschienene (aber davor schon im Internet veröffentlichte) Roman des amerikanischen Schriftstellers Mark Z. Danielewski House of Leaves 
leiten als ,offene Kunstwerke' geradezu und bewusst zu multiplen Rezeptionsmodi und unterschiedlichen Lesarten, wie Hudelist eindrucksvoll zeigt, an.

Dass auch , ,iterarische Mehrsprachigkeit“ vielfältige und zum Teil sehr unterschiedliche Phänomene bezeichnet, zeigt sich in Dominik Sriencs Dissertationsskizze Methodologische Überlegungen zum Gestus literarischen Schreibens bei Florjan Lipuš [168-181]. Der Kärntnerslowenische Autor verfasst seine Werke von Anfang an konsequent auf Slowenisch - nicht nur als literarischen, sondern auch als politisch-gesellschaftlichen Akt des Widerstandes. Srienc' Analyse nähert sich dem Werk Lipuš aus einer bisher vernachlässigten, und zwar aus produktionsästhetischer Perspektive und fokussiert sich auf seine trotz oder gerade aufgrund des Beharrens auf dem ausschließlichen Gebrauch der slowenischen Sprache mehrsprachige literarische Praxis. Die Analyse berücksichtigt biographisch-soziale Aspekte - insbesondere auch die Situation der Kärntner Slowen*innen - und basiert auf unveröffentlichten Materialien aus dem im Klagenfurter Literaturarchiv aufbewahrten Vorlass des Autors.

Schließlich beleuchtet Daniel Wutti in seinem Beitrag Trauma und Mehrsprachigkeit in Gesellschaft und Literatur [182-197] aus psychotraumatologischer Sicht und am Beispiel der Kärntner Slowen*innen, wie sich Sprachregimes und Sprachideologien, die in diglossischen Gesellschaften vorherrschen, auf das Selbstbewusstsein und das Selbstwertgefühl von Sprecher*innen der minorisierten Sprachen auswirken können. Andererseits illustriert Wutti anhand von literarischen Beispielen, wie jedoch gerade Sprache und Mehrsprachigkeit durch Ironie, (schwarzen) Humor und sprachspielerische Schöpfungen helfen können, die Nähe zu ,gewählten Traumata' zu regulieren. Jenseits der konkreten Beispiele zeigt der Autor Perspektiven im Kontext von Sprachminderheiten, aber auch von Flucht und Migration, auf, die eine Überwindung von Hierarchisierungen von Sprachen - und demzufolge der unterschiedlichen Wertschätzung, die deren Sprecher*innen sowie den damit verbundenen idealisierten Kulturvorstellungen entgegengebracht wird anstreben.

Die vorliegende Ausgabe präsentiert somit einen Überblick über die vielfältigen Themen, die im Bereich der Mehrsprachigkeitsforschung an der Alpen-Adria-Universität behandelt werden. Und doch kann dies gezwungenermaßen nur ein kleiner Ausschnitt davon sein: Neben den hier gesammelten Themen und Forschungsbereichen spielt Mehrsprachigkeit auch bei vielen anderen Forscher*innen unserer Universität eine zentrale Rolle, sei es im Bereich der Fachdidaktik und der Lehramtsausbildung, der Gebärdensprachenforschung, der synchronen und diachronen Sprachkontakt- und Minderheitensprachenforschung, der historischen und kulturwissenschaftlichen bzw. kulturanalytischen Forschung, der Migrationsforschung, oder der pädagogischen und erziehungswissenschaftlichen Forschung. Die Bandbreite der Themen und Ansätze, die dabei zum Tragen kom- 
men, ist groß und zeugt davon, wie stark das Interesse dafür an der AAU verankert ist - entsprechend der sowohl national als auch international starken Ausrichtung hin zur Diversität und Vielfalt.

Den Anhang zu dieser Ausgabe bildet ein Wiederabdruck von Das Alpen-Adria Manifest/Il Manifesto dell'Alpe-Adria/Manifest Alpe-Jadran. 1918-2018. War ist over! Se vuoi. $\check{C}$ e hočeš/Wenn du es willst (Wintersteiner et al. 2020), das ein sichtbares Zeichen für Sprachenvielfalt, Mehrsprachigkeit und grenzüberschreitende Zusammenarbeit im Sinne eines friedenstiftenden und friedensbewahrenden Projektes setzt.

\section{Literatur}

Arndt, Susan, Dirk Naguschewski und Robert Stockhammer. Hrsg. 2007. Exophonie. AndersSprachigkeit (in) der Literatur. LiteraturForschung, vol. 3. Berlin: Kadmos.

Aronin, Larissa, Muiris Ó Laoire and David Singleton. 2011. „The multiple faces of multilingualism: Language nominations.“ Applied Linguistics Review 2 (2011): 169-90. https://doi.org/ $10.1515 / 9783110239331.169$.

Auer, Peter and Li Wei, eds. 2007. Handbook of Multilingualism and Multilingual Communication. Handbooks of Applied Linguistics, vol. 5. Berlin, New York: Mouton de Gruyter.

Bachtin, Michael M. [1975] 1979. Die Ästhetik des Wortes. Hrsg. von Rainer Grübel. Übersetzt von Rainer Grübel und Sabine Reese. Frankfurt am Main: Suhrkamp.

Backus, Ad, Durk Gorter, Karlfried Knapp, Rosita Schjerve-Rindler, Jos Swanenberg, Jan D. ten Thije und Eva Vetter. 2013. „Inclusive Multilingualism: Concept, Modes and Implications.“ European Journal of Applied Linguistics 1 (2): 179-215. https://doi.org/10.1515/eujal-20130010 .

Barakos, Elisabeth und Charlotte Selleck. 2019. „Elite Multilingualism: Discourses, Practices, and Debates." Journal of Multilingual and Multicultural Development 40 (5): 361-74. https: //doi.org/10.1080/01434632.2018.1543691.

Bhatia, Tej K. and William C. Ritchie, eds. 2013 [2004]. The Handbook of Bilingualism and Multilingualism. 2. Auflage. Blackwell Handbooks in Linguistics. West Sussex: Wiley-Blackwell.

Bickes, Hans. 2004. „Bilingualismus, Mehrsprachigkeit und mentales Lexikon - evolutionsbiologische, soziokulturelle und kognitionswissenschaftliche Perspektiven“. Fremdsprachen Lehren und Lernen 33: 27-51.

Bickes, Hans und Ute Pauli. 2009. Erst-und Zweitspracherwerb. UTB Sprachwissenschaft 3281. Paderborn: Wilhelm Fink.

Bleichenbacher, Lukas. 2008. Multilingualism in the Movies. Hollywood Characters and Their Language Choices. Tübingen: Francke.

Block, David. 2019. „What on Earth Is 'Language Commodification'?“ In Sloganization in Language Education Discourse. Conceptual Thinking in the Age of Academic Marketization, edited by Barbara Schmenk, Stephan Breidbach and Lutz Küster, 121-41. Bristol: Multilingual Matters. 
Blommaert, Jan. 2010. The Sociolinguistics of Globalization. Cambridge Approaches to Language Contact. Cambridge, New York: Cambridge University Press.

Brugnolo, Furio und Vincenzo Orioles, eds. 2002. Eteroglossia eplurilinguismo letterario, I. Litaliano in Europa, II. Plurilinguismo e letteratura. Lingue, culture e testi, vol. 4/2. Roma: Ed. Il Calamo.

Busch, Brigitta. 2013. Mehrsprachigkeit. UTB Sprachwissenschaft 3774. Wien: Facultas.

Canagarajah, A. Suresh, ed. 2017. The Routledge Handbook of Migration and Language. Routledge Handbooks in Applied Linguistics. London: Routledge.

Carbonara, Valentina and Andrea Scibetta. 2020. „Integrating Translanguaging Pedagogy into Italian Primary Schools: Implications for Language Practices and Children's Empowerment.“ International Journal of Bilingual Education and Bilingualism, April, 1-21. https://doi.org/ 10.1080/13670050.2020.1742648.

Coseriu, Eugenio [1980] 1988 „Historische Sprache“ und ,Dialekt““. In Energeia und Ergon. Sprachliche Variation, Sprachgeschichte, Sprachtypologie; vol.: 1 Schriften von Eugenio Coseriu (1965-1987). Tübinger Beiträge zur Linguistik, vol. 300, hrsg. von Jörn Albrecht, 45-61. Tübingen: Narr.

Costa, James. 2019. „Introduction: Regimes of Language and the Social, Hierarchized Organization of Ideologies." Language \& Communication 66 (2019): 1-5. https://doi.org/10.1016/j. langcom.2018.10.002.

Coulmas, Florian. 2005. „Changing Language Regimes in Globalizing Environments.“ International Journal of the Sociology of Language 175/176: 3-15.

Creese, Angela and Adrian Blackledge, eds. 2018. The Routledge Handbook of Language and Superdiversity. An Interdisciplinary Perspective. Routledge Handbooks in Applied Linguistics. London, New York: Routledge.

Cummins, James. 1981. „The Role of Primary Language Development in Promoting Educational Success for Language Minority Students." In Schooling and Language Minority Students: A Theoretical Framework. Office of Bilingual Bicultural Education, 3-50. Los Angeles: Dissemination Evaluation and Assessment Center.

Cummins, Jim. 2017. „Teaching Minoritized Students: Are Additive Approaches Legitimate?“ Harvard Educational Review 87 (3): 404-25.

De Costa, Peter. 2019. „Elite Multilingualism, Affect and Neoliberalism.“ Journal of Multilingual and Multicultural Development 40 (5): 453-60. https://doi.org/10.1080/01434632.2018. 1543698.

Dembeck, Till und Georg Mein. Hrsg. 2014. Philologie und Mehrsprachigkeit. Beiträge zur neueren Literaturgeschichte, vol. 315. Heidelberg: Universitätsverlag Winter.

Dembeck, Till und Rolf Parr. Hrsg. [2017] 2020. Literatur und Mehrsprachigkeit. Ein Handbuch. 2. Auflage. Tübingen: Narr Francke Attempto.

Dembeck, Till und Anne Uhrmacher. Hrsg. 2016. Das literarische Leben der Mehrsprachigkeit. Methodische Erkundungen. Beiträge zur neueren Literaturgeschichte, vol. 350. Heidelberg: Universitätsverlag Winter.

Dirim, İnci. 2010. „,Wenn man mit Akzent spricht, denken die Leute, dass man auch mit Akzent denkt oder so: Zur Frage des (Neo-)Linguizismus in den Diskursen über die Sprache(n) 
der Migrationsgesellschaft“. In Spannungsverhältnisse: Assimilationsdiskurse und interkulturellpädagogische Forschung, hrsg. von Paul Mecheril, İnci Dirim, Mechtild Gomolla, Sabine Hornberg und Krassimir Stojanov, 91-112. Münster: Waxmann.

Dirim, İnci und Paul Mecheril. 2010. „Die Sprache(n) der Migrationsgesellschaft“. In Migrationspädagogik, hrsg. von Paul Mecheril, María do Mar Castro Varela, İnci Dirim, Annita Kalpaka und Claus Melter, 99-120. Bachelor, Master. Weinheim, Basel: Beltz Verlag.

Duchêne, Alexandre and Monica Heller, eds. 2012. Language in Late Capitalism. Pride and Profit. New York: Routledge.

Fäcke, Christiane und Franz-Joseph Meißner, Hrsg. 2019. Handbuch Mehrsprachigkeits-und Mehrkulturalitätsdidaktik. Tübingen: Narr Francke Attempto.

Franceschini, Rita. 2006. „Mehrsprachigkeit: das Lernpotential von Grenzregionen = Plurilinguismo: il potenziale nelle regioni di confine $=$ Multilingualism: Learning Potential in Border Regions“. In Mehrsprachigkeit in Europa: Erfahrungen, Bedürfnisse, Gute Praxis = Plurilinguismo in Europa: esperienze, esigenze, buone pratiche = Multilingualism across Europe: Findings, Needs, Best Practices 24.-26.08.2006, Bolzano/Bozen, herausgegeben von Andrea Abel, Mathias Stuflesser und Magdalena Putz, 33-41. Bozen: Europäische Akad. Bozen.

Franceschini, Rita. 2009. „The Genesis and Development of Research in Multilingualism. Perspectives for Future Research." In The Exploration of Multilingualism. Development of Research on L3, Multilingualism and Multiple Language Acquisition, edited by Larissa Aronin and Britta Hufeisen, 6: 27-61. AILA Applied Linguistics Series (AALS). Amsterdam, Philadelphia: John Benjamins.

Franceschini, Rita. 2011. „Multilingualism and Multicompetence: A Conceptual View“. The Modern Language Journal 95 (3):344-355. https://doi.org/10.1111/j.1540-4781.2011.01202.x.

Fürstenau, Sara. 2011. „Mehrsprachigkeit als Voraussetzung und Ziel schulischer Bildung“. In Migration und schulischer Wandel: Mehrsprachigkeit, hrsg. von Sara Fürstenau und Mechtild Gomolla, 25-50. Wiesbaden: VS Verlag für Sozialwissenschaften.

García, Ofelia und Wei Li. 2014. Translanguaging. Language, Bilingualism and Education. Palgrave Pivot. New York: Palgrave Macmillan.

Garrido, Maria Rosa. 2017. „Multilingualism and Cosmopolitanism in the Construction of a Humanitarian Elite.“ Social Semiotics 27 (3): 359-369. https://doi.org/10.1080/10350330. 2017.1301800 .

Gerhards, Jürgen. 2010. Mehrsprachigkeit im vereinten Europa. Transnationales sprachliches Kapital als Ressource in einer globalisierten Welt. Wiesbaden: VS Verlag für Sozialwissenschaften.

Gogolin, Ingrid. 1994. Der monolinguale Habitus der multilingualen Schule. Internationale Hochschulschriften. Münster, New York: Waxmann.

Gombos, Georg. 2019. „Zwei- und Mehrsprachigkeit früh fördern. Sprachpädagogische Arbeit in elementarpädagogischen Einrichtungen im Kontext von autochthonen Minderheiten am Beispiel der Kärntner Slowenen“. In Lernraum Mehrsprachigkeit. Zum Umgang mit Minderheitenund Migrationssprachen, hrsg. von Jasmin Donlic, Georg Gombos und Hans Karl Peterlini, 123-143. Klagenfurt: Drava.

Grin, François. 1996. „The Economics of Language: Survey, Assessment und Prospects.“ International Journal of the Sociology of Language 121: 17-44. 
Grin, François. 2005. „Linguistic Human Rights as a Source of Policy Guidelines: A Critical Assessment." Journal of Sociolinguistics 9 (3): 448-460.

Grosjean, François und Ping Li. 2013. The Psycholinguistics of Bilingualism. Hoboken: WileyBlackwell.

Hall, Joan Kelly, An Cheng and Matthew T. Carlson. 2006. „Reconceptualizing Multicompetence as a Theory of Language Knowledge“. Applied Linguistics 27 (2): 220-40. https://doi. org/10.1093/applin/aml013.

Heinemann, Alisha M. B. und İnci Dirim. 2016. „Die sprechen bestimmt (schlecht) über mich“. Sprache als ordnendes Prinzip im Bildungssystem“. In Symbolische Ordnung und Bildungsungleichheit in der Migrationsgesellschaft, hrsg. von Emre Arslan und Kemal Bozay, 199-214. Wiesbaden: Springer.

Heller, Monica, ed. 2007. Bilingualism. A Social Approach. Palgrave Advances in Language and Linguistics. London: Palgrave Macmillan.

Heller, Monica. 2010. „The Commodification of Language“. Annual Review of Anthropology 39 (1): 101-114. https://doi.org/10.1146/annurev.anthro.012809.104951.

Helmich, Werner. 2016. Asthetik der Mehrsprachigkeit. Zum Sprachwechsel in der neueren romanischen und deutschen Literatur. Studia Romanica, vol. 196. Heidelberg: Universitätsverlag Winter.

Irvine, Judith T. 2018. „Regimenting Ideologies“. Language \& Communication 66 (2019): 67-71. https://doi.org/10.1016/j.langcom.2018.10.002.

James, Allan. Hrsg. 2003. Vielerlei Zungen. Mehrsprachigkeit + Spracherwerb + Pädagogik + Psychologie + Literatur + Medien. Klagenfurt: Drava.

Khakpour, Natascha. 2016. „Die Differenzkategorie Sprache. Das Beispiel “Native Speaker”. In Kulturen der Bildung. Kritische Perspektiven auf erziehungswissenschaftliche Verhältnisbestimmungen, hrsg. von Merle Hummrich, Nicolle Pfaff, Inci Dirim und Christine Freitag, 209-20. Wiesbaden: Springer VS.

Kommission der europäischen Gemeinschaften. 2007. „Arbeitsdokument der Kommission. Bericht über die Durchführung des Aktionsplans ,Förderung des Sprachenlernens und der Sprachenvielfalt“'..Letzter Zugriff: 6. Dezember 2020. https://eur-lex.europa.eu/legal-content/DE/ TXT/PDF/?uri=CELEX:52007DC0554.

Kremnitz, Georg. [2004] 2015. Mehrsprachigkeit in der Literatur. Ein kommunikationssoziologischer Überblick. 2. erw. Auflage. Wien: Praesens Verlag.

Leben, Andreas und Alenka Koron. Hrsg. 2019. Literarische Mehrsprachigkeit im österreichischen und slowenischen Kontext. Literarische Mehrsprachigkeit, vol. 2. Tübingen: Narr Francke Attempto.

Lebsanft, Franz und Monika Wingender. Hrsg. 2012. Die Sprachpolitik des Europarats. Die „Europäische Charta der Regional-oder Minderheitensprachen" aus linguistischer und juristischer Sicht. Berlin: De Gruyter.

Lennon, Brian. 2010. In Babel's Shadow. Multilingual Literatures, Monolingual States. Minneapolis, London: University of Minnesota Press. 
Lüdi, Georges. 2011. „Neue Herausforderungen an eine Migrationslinguistik im Zeichen der Globalisierung“. In Sprachen in mobilisierten Kulturen: Aspekte der Migrationslinguistik, hrsg. von Thomas Stehl, 2: 15-38. Mobilisierte Kulturen. Potsdam: Universitätsverlag Potsdam.

MacSwan, Jeff. 2017. „A Multilingual Perspective on Translanguaging.“ American Educational Research Journal 54 (1): 167-201. https://doi.org/10.3102/0002831216683935.

Makoni, Sinfree and Alastair Pennycook, eds. 2007. Disinventing and Reconstituting Languages. Bilingual Education and Bilingualism, vol. 62. Clevedon, Buffalo, Toronto: Multilingual Matters.

Martin-Jones, Marilyn, Adrian Blackledge and Angela Creese, eds. 2012a. The Routledge Handbook of Multilingualism. Routledge Handbooks in Applied Linguistics. London, New York: Routledge.

Martin-Jones, Marilyn, Adrian Blackledge and Angela Creese. 2012b. „Introduction: A Sociolinguistics of Multilingualism for Our Times." In The Routledge Handbook of Multilingualism, edited by Marilyn Martin-Jones, Adrian Blackledge and Angela Creese, 1-26. London; New York: Routledge.

Miklosich, Franz. 1889. Über die Einwirkungen des Türkischen auf die Grammatik der südosteuropäischen Sprachen. Wien: Tempsky.

Müller, Natascha, Tanja Kupisch, Katrin Schmitz und Katja F. Cantone. Hrsg. 2011 [2006]. Einführung in die Mehrsprachigkeitsforschung. Deutsch - Französisch - Italienisch. 3rd revised Editon. Narr Studienbücher. Tübingen: Narr.

Omann, Lisa. 2020. „Der Kärntner Dialekt in der touristischen Kommunikation. Innere Mehrsprachigkeit im alltäglichen Gästekontakt in Kärntner Hotelbetrieben“. Bachelor Thesis, AlpenAdria-Universität Klagenfurt.

Otheguy, Ricardo, Ofelia García and Wallis Reid. 2015. „Clarifying Translanguaging and Deconstructing Named Languages: A Perspective from Linguistics." Applied Linguistics Review 6 (3): 281-307. https://doi.org/10.1515/applirev-2015-0014.

Otheguy, Ricardo, Ofelia García and Wallis Reid. 2019. „A Translanguaging View of the Linguistic System of Bilinguals.“ Applied Linguistics Review 10 (4): 625-51. https://doi.org/10.1515/ applirev-2018-0020.

Paulsrud, BethAnne, Jenny Rosén, Boglárka Straszer and Åsa Wedin, eds. 2017. New Perspectives on Translanguaging and Education. Bilingual Education \& Bilingualism, vol. 108. Bristol: Multilingual Matters.

Pennycook, Alastair. 2010. Language as a Local Practice. Abingdon, New York: Routledge.

Peterlini, Hans Karl. 2019. „Magari mehrsprachig. Das Sprachenmodell Südtirol - von ungenutzten Möglichkeiten, alarmierenden Befunden und jugendlichem Pragmatismus“. In Lernraum Mehrsprachigkeit. Zum Umgang mit Minderheiten- und Migrationssprachen, hrsg. von Jasmin Donlic, Georg Gombos und Hans Karl Peterlini, 197-217. Klagenfurt: Drava.

Piccardo, Enrica and Joëlle Aden. 2014. „Plurilingualism and Empathy: Beyond Instrumental Language Learning." In The Multilingual Turn in Languages Education, edited by Jean Conteh and Gabriela Meier, 234-57. Bristol: Multilingual Matters.

Radaelli, Giulia. 2011. Literarische Mehrsprachigkeit. Sprachwechsel bei Elias Canetti und Ingeborg Bachmann. Deutsche Literatur. Studien und Quellen, vol. 3. Berlin: De Gruyter. 
Riehl, Claudia Maria. 2014. Mehrsprachigkeit. Eine Einführung. Einführung Germanistik. Darmstadt: WBG.

Ritzau, Ursula. 2015. „Learner Language and Polylanguaging: How Language Students' Ideologies Relate to Their Written Language Use." International Journal of Bilingual Education and Bilingualism 18 (6): 660-75. https://doi.org/10.1080/13670050.2014.936822.

Roche, Jörg und Elisabetta Terrasi-Haufe. 2018. Mehrsprachigkeit und Sprachenerwerb. Kompendium DaF/DaZ, vol. 4. Tübingen: Narr Francke Attempto.

Ruiz, Richard. 2010. „Reorienting Language-as-Resource." In International Perspectives on Bilingual Education: Policy, Practice, and Controversy, edited by John E. Petrovic, 155-72. International Perspectives on Educational Policy, Research and Practice. Charlotte: IAP.

Salö, Linus and David Karlander. 2018. „Semilingualism: The Life and Afterlife of a Sociolinguistic Idea." Working Papers in Urban Language \& Literacies 247: 1-14. https://doi.org/10. 13140/RG.2.2.29844.76169.

Schmeling, Manfred und Monika Schmitz-Emans. Hrsg. 2002. Multilinguale Literatur im 20. Jahrhundert. Saarbrücker Beiträge zur vergleichenden Literatur- und Kulturwissenschaft, vol. 18. Würzburg: Königshausen \& Neumann.

Schmitz-Emans, Monika. Hrsg. 2004. Literatur und Vielsprachigkeit. Hermeia, vol. 7. Heidelberg: Synchron.

Schneider, Stefan. 2015. Bilingualer Erstspracherwerb. utb Pädagogik 4348. München, Basel: Ernst Reinhardt Verlag.

Schwieter, John W., ed. 2019. The Handbook of the Neuroscience of Multilingualism. Blackwell Handbooks in Linguistics. Hoboken: Wiley-Blackwell.

Springsits, Birgit. 2015. „»Nein, das kann nur die Muttersprache sein.« Spracherwerbsmythen und Linguizismus". In Sprache und Bildung in Migrationsgesellschaften. Machtkritische Perspektiven auf ein prekarisiertes Verhältnis, hrsg. von Nadja Thoma und Magdalena Knappik, 89108. Kultur und soziale Praxis. Bielefeld: Transcript.

Trabant, Jürgen. 2018. „Befreundung. Für eine gebildete europäische Mehrsprachigkeit“. In Bildung in fremden Sprachen? Pädagogische Perspektiven auf globalisierte Mehrsprachigkeit, hrsg. von Ruprecht Mattig, Miriam Mathias und Klaus Zehbe, 171-193. Pädagogik. Bielefeld: Transcript.

Turner, Marianne and Angel M. Y. Lin. 2017. „Translanguaging and Named Languages: Productive Tension and Desire." International Journal of Bilingual Education and Bilingualism 23 (4): 423-33. https://doi.org/10.1080/13670050.2017.1360243.

Vedovelli, Massimo. 2019. „Nuovi scenari globali per l'Italiano nel mondo“. In Italiano nel mondo. Per una nuova visione, hrsg. von Raffaella Bombi, 181-94. Udine: Forum.

Videsott, Gerda. 2011. Mehrsprachigkeit aus neurolinguistischer Sicht: Eine empirische Untersuchung zur Sprachverarbeitung viersprachiger Probanden. Romanische Sprachen und ihre Didaktik, vol. 30. Stuttgart: ibidem.

Videsott, Gerda. 2020. „Il plurilinguismo dei ladini: aspetti neurolinguistici“. In Manuale di linguistica ladina, hrsg. von Paul Videsott, Ruth Videsott und Jan Casalicchio, 470-479. Manuals of romance linguistics, vol. 26. Boston: De Gruyter. 
Videsott, Gerda, Bärbel Herrnberger, Klaus Hoenig, Edgar Schilly, Jo Grothe, Werner Wiater, Manfred Spitzer and Markus Kiefer. 2010. „Speaking in Multiple Languages: Neural Correlates of Language Proficiency in Multilingual Word Production." Brain \& Language 113: 103112.

Wandruszka, Mario. 1979. Die Mehrsprachigkeit des Menschen. München: Piper.

Weinreich, Uriel. [1953] 1968. Languages in Contact. Findings and Problems. 9. Auflage. The Hague: Mouton.

Wintersteiner, Werner, Georg Gombos und Daniela Gronold. 2008. „15 Thesen zur Mehrsprachigkeit. Anlässlich der Konferenz ,Mehrsprachigkeit, Transkulturalität und Bildung 'Regionalentwicklung des Alpen-Adria-Raums in globaler Perspektive Alpen-Adria-Universität Klagenfurt, 8. - 10. Oktober 2008“. Letzter Zugriff: 6 Dezember 2020. https://www.schulpartner. info/wp-content/myuploads/2009/11/15-thesen-und-5-prinzipien.pdf.

Wintersteiner, Werner et al. 2020. „Alpen-Adria Manifest. Manifesto dell'Alpe-Adria. Manifest Alpe-Jadran. 1918-2018. War is over! Se vuoi. Če hočeš. Wenn du es willst". In Manifest|o Alpe-Adria. Stimmen für eine Europa-Region des Friedens und Woblstands = Vociper una regione europea di pace e prosperità = Glasovi za evropsko regijo miru in blagostanja, hrsg. von Werner Wintersteiner, Mira Miladinovic und Cristina Beretta, 27-62. edition pen, vol. 151. Wien: Löcker.

Wright, Wayne E., Sovicheth Boun and Ofelia García, eds. 2015. The Handbook of Bilingual and Multilingual Education. Blackwell Handbooks in Linguistics. Malden: Wiley-Blackwell. 